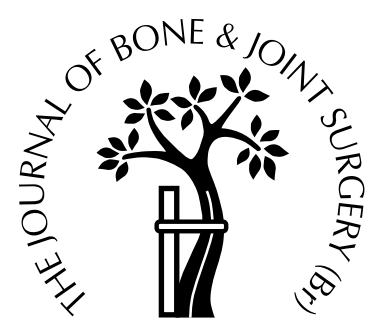

\title{
Function after subtotal scapulectomy for neoplasm of bone and soft tissue
}

\author{
C. L. M. H. Gibbons, R. S. Bell, J. S. Wunder, A. M. Griffin, \\ B. O'Sullivan, C. N. Catton, A. M. Davis \\ From Mount Sinai Hospital, Toronto, Canada
}

$\mathbf{W}^{\mathrm{e}}$ e describe the functional results in 14 patients ( 7 men, 7 women) after subtotal scapulectomy for primary bone and soft-tissue tumours at a specialist musculoskeletal oncology unit. Eight had chondrosarcomas, two Ewing's sarcomas, one aggressive fibromatosis and three soft-tissue sarcomas. The mean follow-up was 52 months (6 to 120). Analysis of residual symptoms and of range and strength of movement by physicians used the Musculoskeletal Tumour Society rating scale (MSTS). Physical disability was measured by the patients using the Toronto Extremity Salvage Score (TESS).

All 14 patients are still alive, two with systemic disease. Nine had more than $80 \%$ of their scapula resected but the glenohumeral joint was preserved in all cases. Eight had full movement and another two achieved $90^{\circ}$ of flexion. The mean functional results were good to excellent in all except three patients (mean MSTS = 71.6 and TESS = 79.9). Two of these three patients had considerable pain as a result of brachial neuropathy.

Scapulectomy gives an excellent functional result if the glenohumeral joint is preserved. The rotator cuff could be removed without a severe functional deficit provided that the deltoid was reattached to the scapular remnant and the trapezius.

J Bone Joint Surg [Br] 1998;80-B:38-42.

Received 21 July 1997; Accepted 1 October 1997

C. L. M. H. Gibbons, MA, FRCS, Consultant Orthopaedic Surgeon Nuffield Orthopaedic Centre, Windmill Road, Headington, Oxford OX3 7LD, UK.

R. S. Bell, MD, FRCS C, Director

J. S. Wunder, MD, FRCS C, Orthopaedic Surgeon

A. M. Griffin, BSc, Research Assistant

A. M. Davis, PhD, Director of Clinical Research

Division of Orthopaedic Surgery and the University Musculoskeletal

Oncology Unit, Suite 476, Mount Sinai Hospital, 600 University Avenue,

Toronto, Canada M5G 1X5.

B. O'Sullivan, FRCP C, Radiation Oncologist

C. N. Catton, FRCP C, Radiation Oncologist

Princess Margaret Comprehensive Cancer Centre, 610 University Avenue, Toronto, Canada M5G 2M9.

Correspondence should be sent to Dr A. M. Davis.

(C)1998 British Editorial Society of Bone and Joint Surgery 0301-620X/98/18183\$2.00
The shoulder is the third most common site for tumours of bone and soft tissues. ${ }^{1}$ The former occur in the proximal humerus, scapula and clavicle in decreasing order of frequency $^{2}$ and the latter most commonly in the deltoid or supraspinatus.

Scapulectomy for tumour was originally described by Syme in $1894 .^{3}$ MRI and CT now allow the accurate assessment of the extent of the lesion, and the effective adjuvant use of irradiation and chemotherapy has extended the indications.

Several studies have described the outcome of treatment and methods of reconstruction after scapulectomy ${ }^{4,5}$ but little attention has been paid to the functional outcome. After total scapulectomy, the function of the upper limb is severely impaired since the patient is left with a hanging shoulder and little rotational control. ${ }^{8,9}$ If, however, all or part of the glenohumeral joint is preserved, good function can be maintained. Kurer et $\mathrm{al}^{7}$ examined postoperative function and correlated the type of resection with the restriction of postoperative movement. O'Connor et $\mathrm{al}^{9}$ described three patients with subtotal scapulectomy who had an average score of 77 on the modified Musculoskeletal Tumour Society scale (MSTS). ${ }^{10}$ The range of movement has been shown to have only low to moderate correlation with the activities of daily living ${ }^{11}$ and evaluation of function is best based on the patients' perception of their own abilities. ${ }^{12,13}$ Meenan ${ }^{14}$ has also shown that the views of the clinician and the patient tend to differ as regards function.

We assessed the effect of subtotal scapulectomy on the function of the upper limb, as judged by the patient, in relation to bone and muscle resection and the method of reconstruction.

\section{Patients and Methods}

We reviewed the records of all patients who had had salvage procedures of the upper limb involving partial or total scapulectomy at the University Musculoskeletal Oncology Unit between 1989 and 1996. Those with a forequarter amputation, shoulder fusion, joint arthroplasty or who had received an allograft reconstruction were excluded, as were patients who had had total scapulectomy and proximal humeral resection as part of the Tikhoff-Linberg 


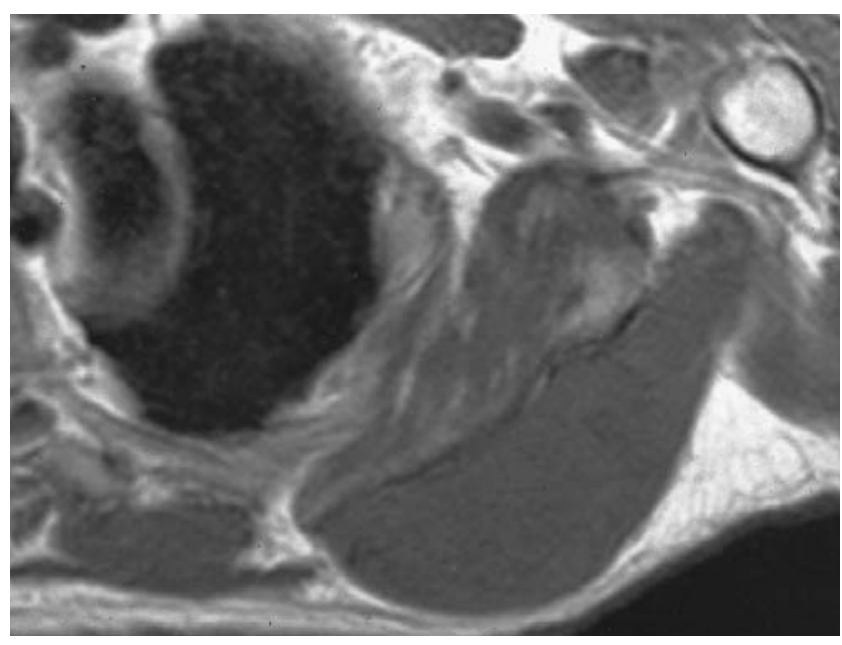

Fig. 1

MRI of a soft-tissue sarcoma involving most of the infraspinatus.

procedure. In all, 14 patients who had had subtotal scapulectomy for primary tumours of bone and soft tissue around the scapula were identified. The seven men and seven women had a mean age of 42 years (16 to 82) at operation.

The clinical records, including the pathological findings and imaging studies, were reviewed. Staging had been by plain radiographs, whole-body technetium bone scans, CT of the chest, CT or MRI of the scapular region and biopsy. The details of the patients, the surgical procedure performed, adjuvant therapy and the postoperative complications were recorded. After operation patients were reviewed at six weeks, three and six months and then at six-monthly intervals. The mean follow-up was 52 months (6 to 120). Two patients were lost to follow-up at 31 and 15 months after operation, one with evidence of local recurrence of disease; their results are included to their latest follow-up.

At review the residual symptoms were recorded, the range of movement noted, the strength in the shoulder assessed using the Medical Research Council grading scale $^{15}$ and the MSTS functional rating system ${ }^{10}$ completed by one clinician. The MSTS allocates scores to pain, overall function, emotional acceptance, positioning of the hand, manual dexterity, and ability to lift, and reflects the clinician's perception of the patient's function. The score ranges from 0 to 100 with 100 representing normal function. Before surgery and at each subsequent review all patients completed a detailed assessment of upper limb function, the Toronto Extremity Salvage Score (TESS). This was developed for those having operations to preserve a limb affected with a tumour ${ }^{16}$ and measures the difficulty experienced in performing routine daily activities. The score ranges from 0 to 100 with higher scores indicative of less disability.

\section{Results}

Clinical presentation. Of the 14 patients, eight had chondrosarcoma, in two arising from osteochondroma of the scapula, and two had Ewing's sarcoma (Figs 1 and 2; Table I). The four soft-tissue tumours requiring partial scapulectomy were two malignant fibrous histiocytomas, one liposarcoma and one case of multiply recurrent, aggressive fibromatosis.

Five patients had had operations before referral to our specialist centre. One with a liposarcoma (case 4) and one with a chondrosarcoma (case 11) had had incomplete excision which required further resection. Two had had multiple resections for local recurrence; one patient with fibromatosis had had eight resections in eight years (case 1) and the other three resections for recurrent chondrosarcoma in five years (case 14). The fifth patient had a recurrence of a

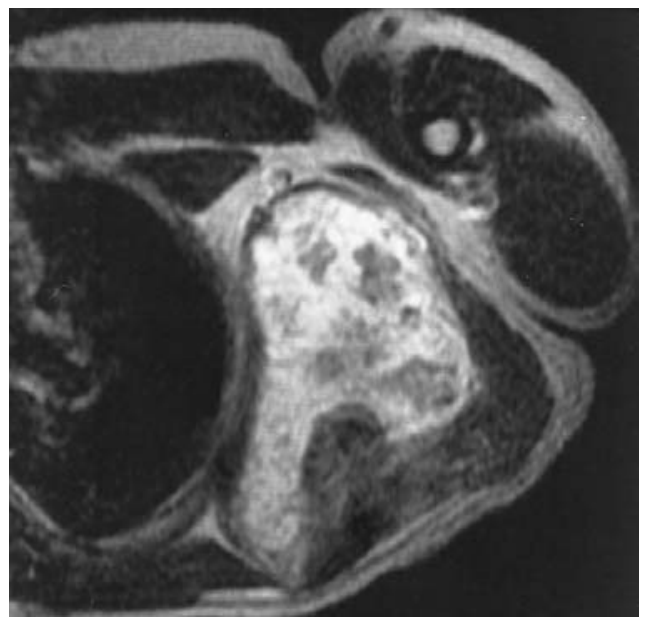

Fig. 2a

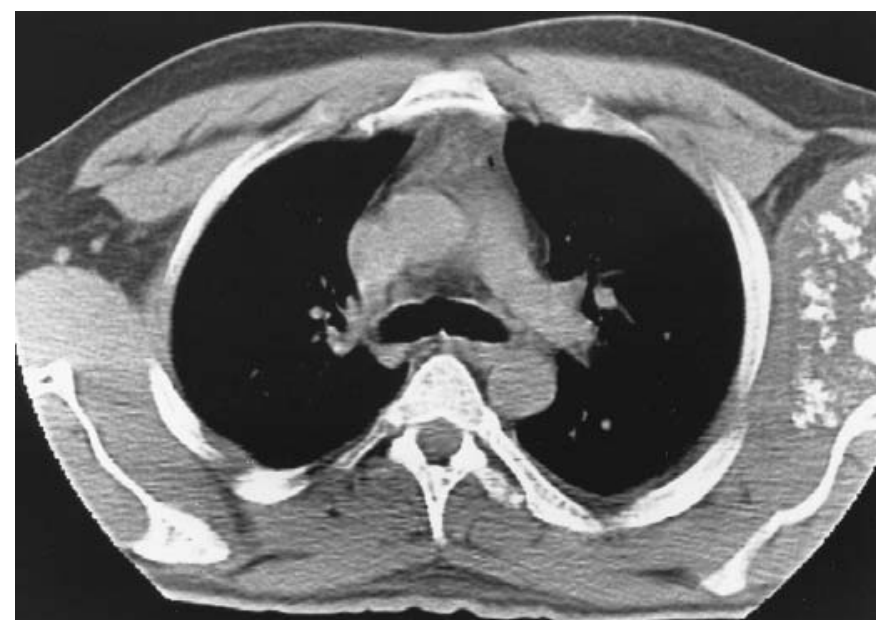

Fig. $2 b$

MRI showing a chondrosarcoma with a large soft-tissue mass extending from the scapula (a) and CT of the same and contralateral site (b). 
Table I. Details of 14 patients who had subtotal scapulectomy

\begin{tabular}{|c|c|c|c|c|c|c|}
\hline Case & Gender & $\begin{array}{l}\text { Age } \\
(\mathbf{y r})\end{array}$ & $\begin{array}{l}\text { Histology* } \\
\text { (grade) }\end{array}$ & $\begin{array}{l}\text { Presenting } \\
\text { status }\end{array}$ & $\begin{array}{l}\text { Scapula } \\
\text { resected }(\%)\end{array}$ & Surgery \\
\hline 1 & M & 20 & Fibromatosis & $\mathrm{LR} \dagger$ & 90 & Chest wall resected \\
\hline 2 & $\mathrm{~F}$ & 29 & $\operatorname{CSA}(2 / 3)$ & Primary & 90 & Excision \\
\hline 3 & $\mathrm{~F}$ & 41 & $\operatorname{CSA}(1 / 3)$ & Primary & 90 & Chest wall resected \\
\hline 4 & $\mathrm{~F}$ & 52 & Liposarcoma $3 / 3$ & LR (nodes) & 90 & Pectoralis major flap \\
\hline 5 & M & 62 & CSA $(2 / 3)$ & Primary & 85 & Excision \\
\hline 6 & M & 39 & CSA $(2 / 3)$ & Primary & 80 & Excision (+ive margin) \\
\hline 7 & $\mathrm{~F}$ & 29 & Ewing's (3/3) & LR & 80 & Excision \\
\hline 8 & M & 72 & $\mathrm{MFH}(3 / 3)$ & Primary & 80 & Excision \\
\hline 9 & M & 33 & $\operatorname{CSA}(2 / 3)$ & Primary & 75 & Excision \\
\hline 10 & M & 82 & $\mathrm{MFH}(3 / 3)$ & Primary & 70 & Latissimus dorsi flap \\
\hline 11 & $\mathrm{~F}$ & 39 & $\operatorname{CSA}(2 / 3)$ & Primary & 50 & Excision \\
\hline 12 & $\mathrm{~F}$ & 37 & CSA $(2 / 3)$ & Primary & 50 & Excision \\
\hline 13 & $\mathrm{~F}$ & 16 & Ewing's $(2 / 3)$ & Primary & 50 & Excision \\
\hline 14 & M & 36 & $\operatorname{CSA}(1 / 3)$ & LR & 80 & Latissimus dorsi flap \\
\hline
\end{tabular}

* CSA, chondrosarcoma; MFH, malignant fibrous histiocytoma

$\dagger$ LR, local recurrence

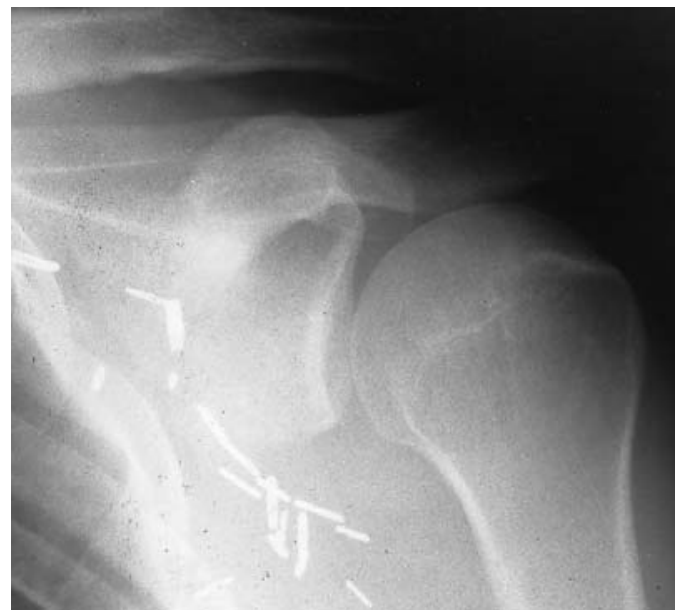

Fig. 3

Plain radiograph showing the residual acromion and coracoid process after $80 \%$ excision of the distal scapula.

Ewing's sarcoma despite complete resection with negative margins six years earlier (case 7).

The tumours involved both bone and soft tissue in all 14 patients. Twelve tumours were high grade and two were low grade. One high-grade tumour, a recurrent liposarcoma, presented with extension into the axillary nodes (case 4).

Tumour excision and reconstruction. All 14 patients had a subtotal scapulectomy. Two patients (cases 1 and 3) had resections of the chest wall, one with a right upper lobectomy, as part of the en-bloc excision of the tumour. All procedures were rated as IIB, according to the classification of Malawar et al, ${ }^{5}$ since all or part of the glenohumeral joint of the scapula was preserved in each case (Fig. 3). Nine had $80 \%$ or more and five had $50 \%$ to $80 \%$ of the scapula resected (Table I).

We performed primary reconstruction of soft tissue in 11 patients. The remnants of the rotator cuff were brought together and reattached to the residual scapula. The serratus anterior was tightened and attached to the remains of the rhomboids and the latissimus dorsi. The posterior deltoid was sutured to the acromion and trapezius so that the remaining scapula was completely covered and stabilised within a muscular envelope.

The remaining three patients (cases 4, 10,14) were left with major defects of muscle and skin and had soft-tissue reconstruction using latissimus dorsi and pectoralis major muscle flaps (Table I).

The surgical margins of resection were grossly clear of tumour in all patients. Microscopic examination of the resected specimens, however, showed positive margins in two. One had further surgery (case 2, margins were revised intraoperatively) and the other (case 6) had postoperative radiotherapy.

Adjuvant therapy. Three patients (cases 4, 7 and 13) received chemotherapy. Both patients with Ewing's sarcoma were treated both before and after operation. The other patient (case 4) had a liposarcoma which had metastasised to the axillary lymph nodes.

Five patients were treated by irradiation including all three with a soft-tissue sarcoma. The patient with multiply recurrent liposarcoma (case 4) had external-beam irradiation $(50 \mathrm{~Gy})$ as well as brachytherapy. The patient with aggressive fibromatosis (case 1) had received irradiation for management of a local recurrence approximately ten years before referral to our centre and was therefore managed by operation alone. One patient (case 6) with a low-grade chondrosarcoma was found to have microscopically positive margins when reviewed histologically and was therefore given postoperative radiotherapy (64 cGy).

Complications. One wound infection requiring surgical debridement occurred in a patient (case 4) who had previously undergone surgery, external-beam irradiation and subsequent brachytherapy. The patient with multiply recurrent fibromatosis treated by surgery and radiotherapy developed brachial neuropathy (case 1). Extensive involvement of the brachial structures with disease was evident in both cases at re-exploration. 
Table II. Functional outcome in 14 patients with subtotal scapulectomy

\begin{tabular}{|c|c|c|c|c|c|c|c|c|}
\hline \multirow[b]{2}{*}{ Case } & \multicolumn{7}{|c|}{ MSTS } & \multirow[b]{2}{*}{ TESS } \\
\hline & Pain & Function & Acceptance & $\begin{array}{l}\text { Hand } \\
\text { positioning }\end{array}$ & Dexterity & Lifting & Total & \\
\hline 1 & 3 & 2 & 5 & 0 & 5 & 0 & 50 & 62 \\
\hline 2 & 5 & 3 & 5 & 5 & 5 & 0 & 77 & 87 \\
\hline 3 & 5 & 3 & 5 & 0 & 5 & 0 & 60 & 95 \\
\hline 4 & 0 & 0 & 0 & 0 & 3 & 0 & 10 & 17 \\
\hline 5 & 0 & 1 & 1 & 0 & 5 & 0 & 23 & 35 \\
\hline 6 & 5 & 5 & 5 & 5 & 5 & 5 & 100 & 98 \\
\hline 7 & 5 & 4 & 5 & 5 & 5 & 4 & 80 & 90 \\
\hline 8 & 5 & 3 & 4 & 5 & 5 & 3 & 83 & 82 \\
\hline 9 & 5 & 5 & 5 & 5 & 5 & 5 & 100 & 100 \\
\hline 10 & 1 & 3 & 5 & 3 & 5 & 1 & 60 & $\mathrm{NA}^{*}$ \\
\hline 11 & 5 & 4 & 5 & 5 & 5 & 4 & 93 & 96 \\
\hline 12 & 4 & 4 & 5 & 5 & 5 & 4 & 90 & 95 \\
\hline 13 & 5 & 5 & 5 & 5 & 5 & 4 & 96 & 95 \\
\hline 14 & 5 & 3 & 5 & 3 & 5 & 3 & 80 & 87 \\
\hline
\end{tabular}

* patient does not speak or read English and was unable to complete the questionnaire

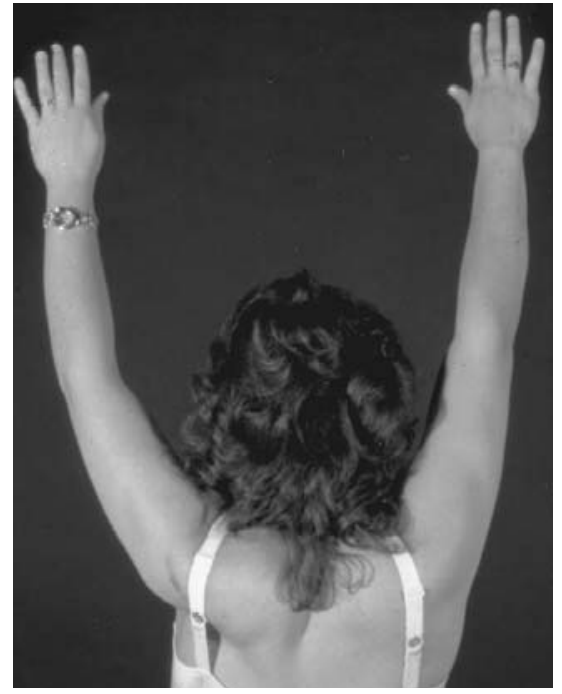

Fig. $4 \mathrm{a}$

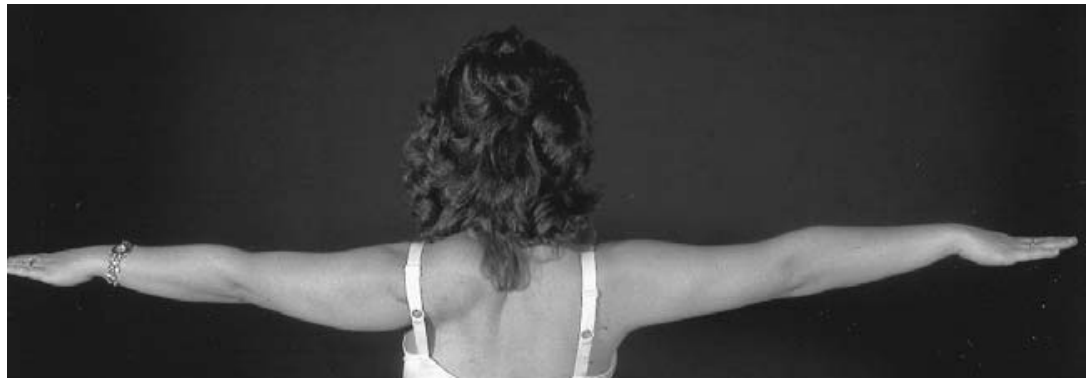

Fig. 4b

Case 2. After resection of $90 \%$ of the scapula there is active flexion (a) and abduction (b).

Oncological outcome. At an average follow-up of 52 months (6 to 120), all patients were alive, although two had systemic disease with local recurrence. One (case 4) with recurrent liposarcoma had involvement of the axillary nodes one year after partial scapulectomy. Further excision was undertaken with dissection of the brachial plexus, capsular repair of the glenohumeral joint and soft-tissue reconstruction using a second vascularised muscle flap. This patient has progressive systemic disease five years after this procedure with a painful brachial neuritis. The second patient (case 7) had presented with local recurrence of a Ewing's sarcoma which subsequently recurred in the surgical scar. Local re-excision was successful but metastases have since developed.

Functional outcome. Five patients complained of pain after surgery, including the two with brachial neuritis. The other three had minor symptoms with one requiring analgesia and the remaining two complaining of discomfort when lying on the shoulder.

Eight patients (cases 2, 6 to 9 and 11 to 13) had full elevation of the shoulder and another two (cases 10 and 14) achieved $90^{\circ}$ of flexion. Of the remaining four patients, three (cases 1, 3 and 5) had $60^{\circ}$ of flexion while one (case 4) with a brachial plexus neuropathy had minimal active movement. Eleven patients had full movement and strength in the elbow and 12 had a normal hand. Deficiency of the elbow and hand occurred in the patients with involvement of the brachial plexus.

The mean functional score as rated by the clinician on the $\operatorname{MSTS}^{10}$ was 71.6 (10 to 100) with deficits noted in positioning of the hand in six of the 14 patients. The patient's own assessment of disability as measured by the TESS had a mean score of 79.9 (17 to 100). Two patients (cases 4 and 5), one with brachial plexus neuropathy (case 4), had very low scores on both the MSTS and TESS (Table II). The patient with aggressive fibromatosis (case 1), who had experienced multiple recurrences and operations, scored 50 and 62 on the MSTS and TESS respectively (Table II).

Twelve patients returned to the same level of activity or 
occupation after operation. Three heavy manual workers reported no restrictions with work. One, with $90 \%$ resection of the scapula, was able to return to competitive volleyball (Fig. 4).

As long as the glenohumeral joint is preserved, the amount of the remaining scapula removed does not appear to affect the functional outcome. The nine patients with $80 \%$ or more of the scapula excised had a mean TESS score of $72( \pm 28.6)$.

\section{Discussion}

After total scapulectomy the function of the upper limb is severely impaired, but good use can be maintained when all or part of the glenohumeral joint is preserved. Subtotal scapulectomy is preferable to ablation of the joint if it can be achieved with good surgical margins.

The most significant functional deficits noted in our series were in three patients (cases 1, 4 and 5) who had $80 \%$ or more of the scapula excised, but they also had multiple local recurrences or significant complications such as brachial neuropathy.

Although subtotal resection of the scapula may result in reduced shoulder flexion and overhead strength, most of our patients reported minimal disability in day-to-day activities. Most activities involving the arm occur in the forward plane just above waist level so that the effect of impairment of overhead use is small. Often, the other arm can help to compensate.

The scapula can be considered as a sophisticated sesamoid bone, and good shoulder function is possible when the soft-tissue envelope can be reconstructed. If the joint capsule must be resected with the tumour, a fascial graft may be used to close and reinforce the defect. In three of our patients muscle flaps were used to provide a vascularised bed over the exposed bone and joint which was suitable for split-thickness skin grafting. Two patients who had latissimus dorsi flaps had excellent functional results with nearly full movement and no pain. The patient who had a pectoralis major flap after scapulectomy for recurrent liposarcoma had a poor functional result because of brachial neuritis.

The rotator cuff can be excised completely; if all or part of the deltoid is preserved and reattached to trapezius and the glenoid remnant, a nearly full range of abduction, flexion and forward elevation can be achieved. The rotator cuff stabilises and centres the glenohumeral joint; careful repair and reinforcement of the cuff to the scapular remnant can maintain glenohumeral stabilisation.

The deltoid can be excised with no significant loss of motion. Markhede et al $^{6}$ showed only a modest functional deficit and no limitation in passive movement in five patients who had either complete removal of the deltoid or complete denervation after resection of a soft-tissue tumour. This can be explained by either compensatory hypertrophy of the remaining rotator cuff, biceps and pectoralis major, or by the remaining muscles contributing increased force after defunctioning of the prime mover of the joint, the deltoid. Kurer et $\mathrm{al}^{7}$ also described good to excellent function after scapulectomy with preservation of the joint. Supraspinous resection provided a reduction in flexion and abduction and an infraspinous resection reduced external rotation. After total scapulectomy there is considerable limitation of movement.

Subtotal scapulectomy gives an excellent functional result if all or part of the glenohumeral joint can be preserved. In our series resection of $80 \%$ of the scapula had only a modest effect on function. The rotator cuff could be excised without significant functional deficit provided that the deltoid was reattached to the scapular remnant and to the trapezius.

No benefits in any form have been received or will be received from a commercial party related directly or indirectly to the subject of this article.

References

1. Enneking WF. Musculoskeletal tumor surgery. Vol. 1. New York: Churchill Livingstone, 1983.

2. Creighton JJ Jr, Peimer CA, Mindell ER, et al. Primary malignant tumours of the upper extremity: retrospective analysis of 126 cases. $J$ Hand Surg Am 1985;10:805-14.

3. Syme J. Excision of the scapula. Edinburgh: Edmonston and Douglas, 1864.

4. Papaioannou AN, Francis KC. Scapulectomy for the treatment of primary malignant tumours of the scapula. Clin Orthop 1965;41: $125-32$.

5. Malawer MM, Meller I, Dunham WK. A new surgical classification system for shoulder-girdle resections: analysis of 38 patients. Clin Orthop 1991;267:33-44

6. Markhede G, Monastyrski J, Stener B. Shoulder function after deltoid muscle removal. Acta Orthop Scand 1985;56:242-4.

7. Kurer MHJ, Bayley JI, Kemp HBS, Pringle JAS. Movement of the shoulder after resection of a tumor of the scapula. J Bone Joint Surg [Am] 1988;70-A:843-7.

8. Frassica FJ, Unni KK, Beabout JW, Sim FH. Dedifferentiated chondrosarcoma: a report of the clinicopathological features and treatment of seventy-eight cases. J Bone Joint Surg [Am] 1986;68-A: 1197-205.

9. O'Connor MI, Sim FH, Chao EYS. Limb salvage for neoplasms of the shoulder girdle: intermediate reconstructive and function results. J Bone Joint Surg [Am] 1996;78-A:1872-88.

10. Enneking WF, Dunham W, Gebhardt MC, Malawar M, Pritchard DJ. A system for the functional evaluation of reconstructive procedures after surgical treatment of tumours of the musculoskeletal system. Clin Orthop 1993;286:241-6.

11. Badley EM, Lee J. Impairment, disability and the ICIDH Model III: underlying disease, impairment, and disability. Int Rehabil Med 1987; 8:174-81.

12. Nayfield SG, Ganz PA, Moinpour CM, Cella DF, Hailey BJ. Report from a National Cancer Institute (USA) workshop on quality of life assessment in cancer clinical trials. Qual Life Res 1992;1:203-10.

13. Rineberg BA. A call to leadership: the role of orthopaedic surgeons in musculoskeletal outcomes research. J Bone Joint Surg [Am] 1990; 72-A:1439-40.

14. Meenan RF. The AIMS approach to health status measurement: conceptual background and measurement properties. J Rheumatol 1982;9:785-8

15. Medical Research Council. Aids to examination of the peripheral nervous system. Memorandum No. 45, London. HMSO, 1976.

16. Davis AM, Wright JG, Williams JI, et al. Development of a measure of physical function for patients with bone and soft tissue sarcoma Qual Life Res 1996;5:508-16. 